'Unidad de Coloproctología, Departamento de Cirugía Digestiva. Pontificia Universidad Católica de Chile, Santiago, Chile. aInterno Escuela de Medicina. Pontificia Universidad Católica de Chile.

Los autores no tienen conflictos de intereses ni fuentes de financiamiento que reportar.

Recibido el 29 de julio de 2014, aceptado el 28 de enero de 2015

Correspondencia a: Dr. Gonzalo Urrejola Schmied Marcoleta 350, patio interior. Santiago, Chile. Teléfono-Fax: (+562) 23543462 giurrejola@uc.c

\section{Ileostomía en asa derivativa y lavado colónico intraoperatorio como alternativa de tratamiento en un caso de colitis grave por Clostridium difficile}

\author{
FELIPE QUEZADA ${ }^{1}$, RICHARD CASTILLO ${ }^{1}$, CONSTANZA VILLALÓN ${ }^{1}$, \\ JOSÉ MIGUEL ZÚÑIGA, CARLA MANTEROLA ${ }^{a}$, MARÍA ELENA MOLINA ${ }^{1}$, \\ FELIPE BELLOLIO ${ }^{1}$, GONZALO URREJOLA ${ }^{1}$

\section{Treatment of a severe Clostridium difficile infection with colonic lavages. Report of one case}

\begin{abstract}
A loop ileostomy with intraoperative anterograde colonic lavage has been described as an alternative to colectomy in the management of cases of Clostridium difficile infection refractory to medical treatment. We report a 69 years old diabetic women admitted with a septic shock. An abdominal CAT scan showed a pan-colitis that seemed to be infectious. A polymerase chain reaction was positive for Clostridium Difficile. Due to the failure to improve after full medical treatment, a derivative loop ileostomy and intra-operatory colonic lavage were performed, leaving a Foley catheter in the proximal colon. In the postoperative period, anterograde colonic instillations of Vancomycin flushes through the catheter were performed every 6 hours. Forty eight hours after surgery, the patient improved. A colonoscopy prior to discharge showed resolution of the pseudomembranous colitis.
\end{abstract}

(Rev Med Chile 2015; 143: 668-672)

Key words: Clostridium difficile; Colectomy; Ileostomy; Vancomycin.

\footnotetext{
L
} a infección por Clostridium difficile (ICD) es la causa de diarrea más frecuente asociada $\checkmark$ a atención de salud ${ }^{1}$, con una morbilidad y mortalidad que ha ido en aumento tanto a nivel nacional ${ }^{2-3}$ como internacional $^{4-6}$. C. difficile es una bacteria Gram (+) formadora de esporas, que produce exotoxinas que son nocivas para la mucosa colónica y gatillan una respuesta inflamatoria tanto local como sistémica ${ }^{7}$.

El diagnóstico se basa en la presencia de un cuadro clínico de diarrea moderada a severa o íleo, asociado a la confirmación microbiológica de la toxina de $C$. difficile y la presencia de $C$. difficile productor de toxina en deposiciones, o el hallazgo de una colitis pseudomembranosa en el estudio colonoscópico y/o histopatológico ${ }^{8}$. En la actualidad no existe un método estándar para la detección de $C$. difficile, siendo recomendado incluso el uso de algoritmos diagnósticos en dos o tres etapas. Últimamente, el uso de métodos de amplificación de ácidos nucleicos, como una reacción de polimerasa en cadena para detección de cepas tóxicas de $C$. difficile, ha sido recomendado para la confirmación diagnóstica de la ICD dado su alta sensibilidad y especificidad ${ }^{9}$.

El espectro de manifestaciones clínicas de la ICD va desde pacientes portadores asintomáticos hasta aquellos que presentan un cuadro grave que puede asociarse a riesgo vital y requerir de un manejo quirúrgico. 
La indicación quirúrgica en pacientes con una ICD grave no está clara y ha sido reservada para pacientes con signos de perforación o megacolon tóxico ${ }^{10}$, siendo la colectomía total con ileostomía terminal el procedimiento de elección. Esta intervención, en este contexto, se asocia a una mortalidad de $50-80 \%$, una morbilidad importante y una baja tasa de reconstitución de tránsito ${ }^{7,11}$.

Una alternativa menos mórbida y con buenos resultados iniciales ha sido la propuesta por Zuckerbraun et al, quienes en pacientes con colitis grave por $C$. difficile realizaron una ileostomía de descarga con lavado colónico intraoperatorio. Los resultados preliminares de esta técnica mostraron una disminución de la tasa de mortalidad hasta $19 \%$, se evitó la colectomía en $93 \%$ de los pacientes de la serie, y la tasa de reconstitución de tránsito fue de $79 \%{ }^{12}$.

El presente caso clínico corresponde a la descripción del primer paciente tratado en nuestra institución con este procedimiento.

El objetivo de este artículo es describir la técnica quirúrgica de ileostomía de descarga y lavado colónico intraoperatorio para el manejo de ICD grave, a propósito de un caso clínico, como alternativa terapéutica para evitar una colectomía en estos pacientes.

\section{Caso clínico}

Mujer de 69 años, con antecedentes de Diabetes Mellitus Tipo 2 y nefropatía médica que fue hospitalizada por un cuadro de pielonefritis aguda, recibiendo terapia con amikacina. Una semana posterior a su alta llegó al servicio de urgencia con un cuadro compatible con un shock séptico grave, ingresando a la unidad de cuidados intensivos, con requerimientos de ventilación mecánica y uso de drogas vasoactivas. De los exámenes generales destacaba leucocitosis de 29.400 células $/ \mathrm{mm}^{3}$ con desviación izquierda, proteína $C$ reactiva de $34,1 \mathrm{mg} / \mathrm{dL}$ (VN $<5 \mathrm{mg} / \mathrm{dL}$ ), acidosis metabólica y falla renal en anuria. Se inició cobertura antibiótica intravenosa de amplio espectro (vancomicina-metronidazol-meropenem-amikacina) y se realizó una tomografía axial computada que evidenció una pancolitis de aspecto inflamatorio-infeccioso, sin evidencias de megacolon ni perforación. Evolucionó hemodinámicamente inestable, mal perfundida y con deposiciones líquidas abundantes (1.000-1.500 ml/día) con una PCR para $C$. difficile positiva, altamente sugerente de cepa NAP1/BI/027, por lo que se indicó vancomicina por vía enteral y enemas. La paciente evolucionó con aumento en los requerimientos de drogas vasoactivas, y hemofiltración de alto volumen. Al quinto día de hospitalización presentó mayor deterioro hemodinámico y aumento de drogas vasoactivas (noradrenalina hasta $0,7 \mathrm{mcg} / \mathrm{kg} / \mathrm{min}$ ), por lo que fue evaluada por el equipo de coloproctología, decidiendo su resolución quirúrgica.

Técnica quirúrgica: se realizó una laparotomía periumbilical de unos 5 centímetros, encontrando moderada cantidad de líquido libre claro y una pancolitis extensa sin compromiso isquémico ni perforación. Se decidió realizar un lavado colónico intraoperatorio e ileostomía en asa derivativa, para lo cual se extrajo un asa de íleon distal para confeccionar la ileostomía. Se realizó una enterotomía pequeña en el sitio de maduración del asa, avanzando hacia distal una sonda Foley 24F (Figura 1) e inflando el balón dentro del ciego con asistencia manual a través de la laparotomía umbilical. Se realizó un lavado colónico anterógrado con 8 litros de polietilenglicol al 7,5\% por de la sonda Foley, recolectando las deposiciones a través de una sonda Pezzer por el ano, hasta dar salida a líquido claro. Se procedió al cierre de la laparotomía y se maduró la ileostomía en asa como muestra la Figura 2. La paciente salió estable de pabellón, con la indicación de lavados anterógrados con vancomicina cada 6 h por la sonda Foley (suero Ringer Lactato 500 $\mathrm{ml} /$ vancomicina $500 \mathrm{mg}$ a pasar en 1 hora) y metronidazol intravenoso.

A las $48 \mathrm{~h}$ post operatorias la paciente fue extubada, se suspendió el uso de drogas vasoactivas e inició diuresis espontánea. Se realimentó con líquidos al tercer día, y tuvo un postoperatorio sin incidentes, salvo por una infección del tracto urinario que fue tratada. Se realizó un control colonoscópico al alta que evidenció resolución de la colitis pseudomembranosa.

La paciente fue dada de alta sin incidentes y sin evidencias de recurrencia de enfermedad en los controles ambulatorios, decidiéndose cierre diferido de la ileostomía. 


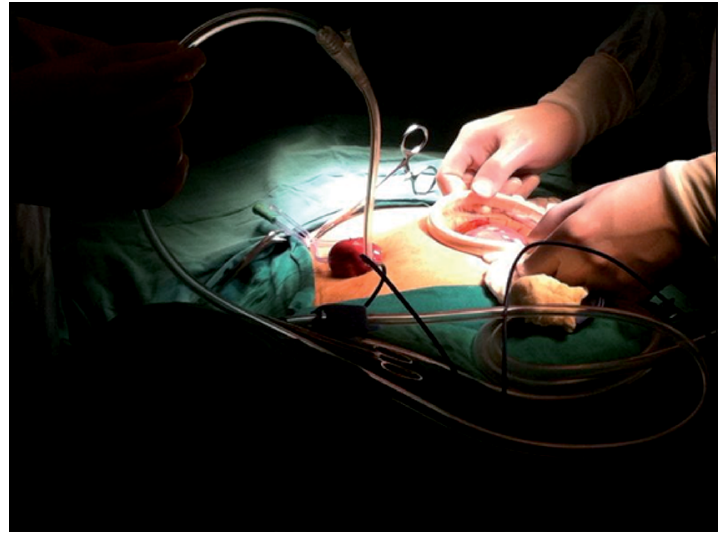

Figura 1. Una vez instalada la sonda Foley a través de enterotomía, se insufló el cuff distal a válvula ileocecal, corroborando su posición a través de la laparotomía.

\section{Discusión}

Este caso es, a nuestro entender, la primera experiencia comunicada en Chile del uso del lavado colónico e ileostomía en asa derivativa para el tratamiento de ICD grave.

La epidemiología de $C$. difficile ha cambiado rápidamente desde el uso amplio de antibióticos ${ }^{13}$. Durante la última década, la ICD se ha hecho más frecuente, presentándose además con episodios de mayor gravedad y refractarios al tratamiento habitual $^{12,13}$. Este viraje se ha atribuido, en parte, a los brotes epidémicos de la cepa hipervirulenta NAP1/ $\mathrm{BI} / 027$, caracterizada por una mayor producción de toxinas, resistencia a las quinolonas, polimorfismos en un gen que disminuye la expresión de toxinas, y la presencia de un gen codificador de una toxina binaria adicional ${ }^{14,15}$.

A pesar de observar una mayor frecuencia de crisis graves, no existe un sistema de graduación actual de gravedad validado y uniforme. La sociedad americana de enfermedades infecciosas (IDSA) define una ICD grave, cuando presenta más de 15.000 leucocitos $/ \mathrm{mm}^{3}$ o un aumento de la creatinina basal en 1,5 veces sobre el valor basal ${ }^{10}$. La sociedad europea de microbiología suma a estos criterios, una concentración de albúmina sérica menor a 3,0 mg/dl y considera a pacientes con alto riesgo de desarrollar una ICD grave a aquellos mayores de 65 años, con comorbilidades importantes, ingresados a una unidad de tratamiento intensivo, inmunodeficientes o con coagulopatías ${ }^{16-18}$.

De esta misma forma, no existe consenso en

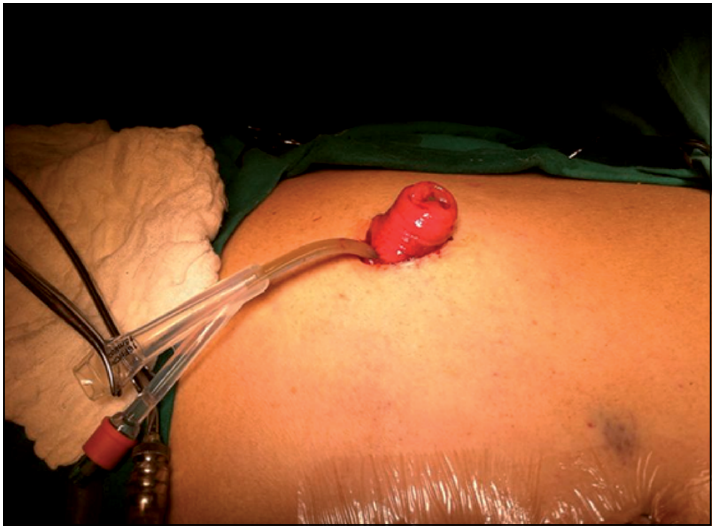

Figura 2. Maduración de ileostomía posterior a lavado y cierre de laparotomía, dejando sonda Foley dentro del polo cecal para realizar instilación anterógrada de vancomicina.

la indicación quirúrgica en pacientes con ICD graves. Las guías clínicas americanas recomiendan fuertemente la evaluación quirúrgica precoz y considerar la cirugía en pacientes con requerimiento de drogas vasoactivas, disfunción orgánica en contexto de sepsis, compromiso de conciencia, leucocitosis sobre 50.000 células $/ \mathrm{mm}^{3}$, lactato sobre $5 \mathrm{mmol} / \mathrm{l}$; o falla de terapia médica después de 5 días $^{9}$. Una revisión sistemática reciente suma a estas características la edad sobre 65 años y la presencia de criterios radiográficos como perforación o isquemia ${ }^{17}$.

La alternativa quirúrgica reportada en este caso fue inicialmente descrita por Zuckerbraun et al, con una mortalidad a 30 días de $19 \%$ y sólo 3 pacientes de la serie requirieron una colectomía total posteriormente. En este contexto, los autores de dicho trabajo proponen un nuevo sistema de graduación aún no validado y basado en parámetros clínicos, de laboratorio e imagenológicos, cuya implementación estaría destinada a fomentar una evaluación e intervención quirúrgica precoz ${ }^{12,17}$.

La indicación quirúrgica en nuestro caso estuvo determinada por criterios clínicos y ausencia de mejoría de la paciente, más que por criterios de laboratorio o radiológicos. La cirugía realizada fue técnicamente sencilla. Se prefirió un abordaje abierto para evaluar directamente el compromiso colónico y realizar el lavado con control directo de la canulación de la sonda hacia el polo cecal para evitar iatrogenias. La infusión de la vancomicina, tanto del lavado como posteriormente, 
fue sencilla, sin inconvenientes durante su administración.

\section{Conclusión}

La ileostomía en asa con lavado colónico intraoperatorio tiene la ventaja de una menor morbilidad asociada que la colectomía total, con resultados al menos similares en cuanto a manejo de la colitis refractaria. Podría ofrecerse en fases más precoces de la enfermedad, en pacientes que no presenten signos de perforación ni necrosis colónica, lo que debería establecerse por parámetros clínicos, de laboratorio e imagenológicos ${ }^{19,20}$.

Creemos que la técnica de la ileostomía con lavado estaría indicada en aquellos pacientes que a pesar del uso de terapia antibiótica estándar (vancomicina + metronidazol a dosis plena), presentan un progresivo deterioro. Esta alternativa quirúrgica, menos mórbida, debería plantearse antes que el paciente presente evidencia de falla multiorgánica o signos de peritonitis, ambos elementos que normalmente están presentes al indicar la colectomía total. Es, por lo tanto, indispensable una evaluación multidisciplinaria precoz en la evolución de la ICD para una adecuada selección de los pacientes susceptibles a recibir este tipo de tratamiento.

\section{Referencias}

1. Fekety R, Shah AB. Diagnosis and treatment of Clostridium difficile colitis. JAMA 1993; 269: 71-5.

2. Gardilcic M, Fica A, Chang M, Llanos C, Luzoro A. Diarrea asociada a Clostridium difficile en un hospital de adultos. Estudio descriptivo. Rev Chilena Infectol 2000; 17: 307-12.

3. Sociedad Chilena de Infectología. Informe: infecciones por Clostridium difficile en el Hospital de Urgencia Asistencia Pública (HUAP) Dr. Alejandro del Río de Santiago, Chile. Rev Chilena Infectol 2012; 29: 531-8.

4. Pépin J, Valiquette L, Alary ME, Villemure P, Pelletier A, Forget $\mathrm{K}$, et al. Clostridium difficile-associated diarrhea in a region of Quebec from 1991 to 2003: a changing pattern of disease severity. CMAJ 2004; 171: 466-72.

5. Loo VG, Poirier L, Miller MA, Oughton M, Libman MD, Michaud S, et al. A predominantly clonal multi-institutional outbreak of Clostridium difficile-associated diarrhea with high morbidity and mortality. N Engl
J Med 2005; 353: 2442-9.

6. Muto CA, Pokrywka M, Shutt K, Mendelsohn AB, Nouri $\mathrm{K}$, Posey K, et al. A large outbreak of Clostridium diffici$l e$-associated disease with an unexpected proportion of deaths and colectomies at a teaching hospital following increased fluoroquinolone. Infect Control Hosp Epidemiol 2005; 26: 273-80.

7. Dallal RM, Harbrecht BG, Boujoukas AJ, Sirio CA, Farkas LM, Lee KK, et al. Fulminant Clostridium difficile: an underappreciated and increasing cause of death and complications. Ann Surg 2002; 235: 363-72.

8. Bauer MP, McFee RB, Kuijper EJ, Abdelsayed GG, van Dissel JT. European Society of Clinical Microbiology and Infectious Diseases (ESCMID): treatment guidance document for Clostridium difficile infection (CDI). Clin Microbiol Infect 2009; 15: 1067-79.

9. Surawicz CM, Brandt LJ, Binion DG, Ananthakishnan AN, Curry SR, et al. Guidelines for diagnosis, treatment, and prevention of Clostridium difficile infections. Am J Gastroenterol 2013; 108 (4): 478-98.

10. Cohen SH, Gerding DN, Johnson S, Kelly CP, Loo VG, McDonald LC, et al. Clinical practice guidelines for Clostridium difficile infection in adults: 2010 update by the society for healthcare epidemiology of America (SHEA) and the infectious diseases society of America (IDSA). Infect Control Hosp Epidemiol 2010; 31: 43155.

11. Longo WE, Mazuski JE, Virgo KS, Lee P, Bahadursingh AN, Johnson FE. Outcome after colectomy for Clostridium difficile colitis. Dis Colon Rectum 2004; 47: 1620-6.

12. Neal MD, Alverdy JC, Hall DE, Simmons RL, Zuckerbraun BS. Diverting loop ileostomy and colonic lavage: an alternative to total abdominal colectomy for the treatment of severe, complicated Clostridium difficile associated disease. Ann Surg 2011; 254: 423-7.

13. Bartlett JG. Narrative review: the new epidemic of Clostridium difficile-associated enteric disease. Ann Intern Med 2006; 145: 758-64.

14. McDonald LC, Killgore GE, Thompson A, Owens RC Jr, Kazakova SV, Sambol SP, et al. An epidemic, toxin gene-variant strain of Clostridium difficile. $\mathrm{N}$ Engl J Med 2005; 353: 2433-41.

15. Warny M, Pepin J, Fang A, Killgore G, Thompson A, Brazier J, et al. Toxin production by an emerging strain of Clostridium difficile associated with outbreaks of severe disease in North America and Europe. Lancet 2005; 366: 1079-84.

16. Bhangu A, Nepogodiev D, Gupta A, Torrance A, Singh P. Systematic review and meta-analysis of outcomes following emergency surgery for Clostridium difficile 
colitis. Br J Surg 2012; 99 (11): 1501-13.

17. Lee DY, Chung EL, Guend H, Whelan RL, Wedderburn RV, Rose KM. Predictors of mortality after emergency colectomy foe Clostridium difficile colitis: an analysis of ACS-NSQIP. Ann Surg 2014; 259 (1): 148-56.

18. Klobuka AJ, Markelov A. Current status of surgical treatment for fulminant Clostridium difficile colitis. World J Gastrointest Surg 2013; 5 (6): 167-72.
19. Brown CJ, Butros M, Morris A, Divino CM. CAGS and ACS evidence based reviews in surgery. Is a diverting loop ileostomy and colonic lavage an alternative to colectomy for the treatment of severe Clostridium difficile-associated disease? Can J Surg 2014; 57 (3): 214-6.

20. Bannura G, Ross G, Gabler F, Esperguel C. Colitis fulminante asociada a Clostridium difficile. Rev Chil Cir 2012; 64 (4): 383-6. 